\title{
Perched facets diagnosed in a neurologically intact patient 5 weeks after a fall
}

\author{
Brian Reed Curtis ${ }^{1} \cdot$ Eric Curtis ${ }^{2}$
}

Received: 8 February 2017 / Accepted: 8 March 2017/Published online: 16 March 2017

(C) SIMI 2017

\section{Case presentation}

A 72-year-old man presented to our hospital's emergency department (ED) for evaluation of cervical spine instability. Five weeks prior to presentation to our ED, he was trimming a tree, and fell 8-10 ft. The fall rendered him unconscious, and he was taken to an outside hospital where he was evaluated and discharged in a hard collar with scheduled imaging and neurosurgical follow-up in 5 weeks. The patient had cervical spine radiographs obtained prior to his neurosurgical appointment. These images showed perched facets of $\mathrm{C} 4$ on $\mathrm{C} 5$. The patient was in our vicinity, and was directed immediately to the ED of our hospital. He had neck pain at presentation, but no neurological symptoms. He underwent MRI of the cervical spine, which better characterized the ligamentous extent of the hyperflexion injury. He was evaluated by neurosurgery, and underwent open reduction and internal fixation (ORIF) due to cervical spine instability. Anterior cervical diskectomy and fusion (ACDF) with lordotic allograft spacer and plate and screw fixation was performed. He did well postoperatively, and was discharged in a hard collar. He has no neurologic deficits or symptoms.

Brian Reed Curtis

brian.curtis@jefferson.edu

1 Department of Radiology, Thomas Jefferson University Hospital, 132 South 10th Street, Philadelphia, PA 19107, USA

2 Virginia Commonwealth University School of Medicine, Richmond, VA, USA

\section{Imaging findings}

See Figs. 1 and 2.

\section{Discussion}

Cervical spine trauma is a significant cause of morbidity and mortality. There are several mechanisms that lead to distinct patterns of injury. Bilateral facet dislocation injuries are thought to arise from a flexion-distraction mechanism [1]. With flexion and distraction of the cervical spine, the facet joints are exposed to disruptive tensional stress that leads to perching or jumping of the inferior articular processes of the superior vertebrae onto the superior articular processes of the inferior vertebrae. This tensional stress can also exceed the tensile capacity of the posterior longitudinal ligament and posterior ligamentous complex resulting in their disruption. Anteriorly, flexiondistraction injuries cause compressive stress that can result in vertebral body and disk compression injuries [2]. All of these findings are present in our case (Figs. 1 and 2).

Carrino et al. retrospectively identified patients with bilateral perched or jumped facets, and find $97 \%$ of the patients have injury to the interspinous and supraspinous ligaments, $90 \%$ have injury to the ligamentum flavum, $90 \%$ have disruption of the intervertebral disk, $40 \%$ have posterior longitudinal ligament disruption, and $27 \%$ have anterior longitudinal ligament disruption [3]. Indeed, MRI shows our patient had perched facets at C4-C5 with bowing of the PLL, injury to the interspinous ligaments and ligamentum flavum and disruption of the intervertebral disk with posterior extrusion. Interestingly, the PLL was found to be disrupted at surgery, meaning MRI was false negative for this finding. Carrino et al. show false-negative 


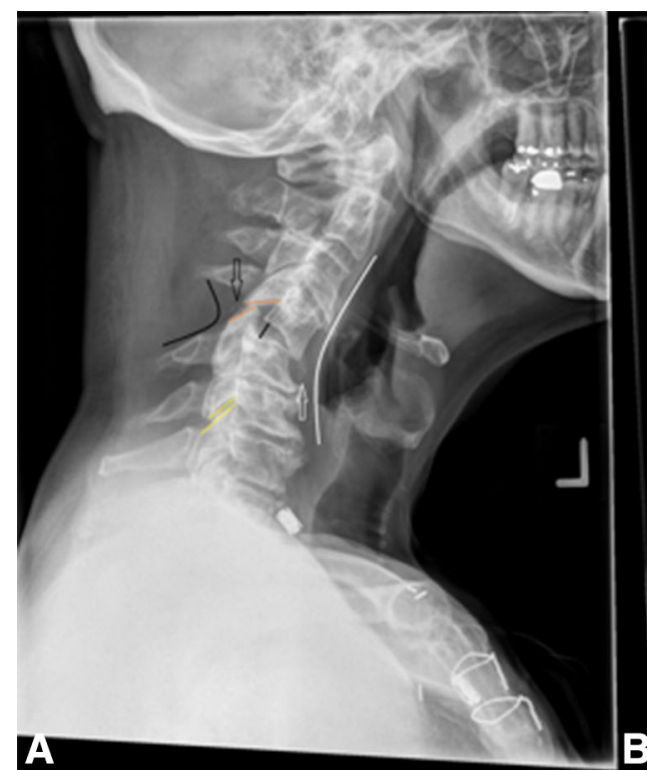

Fig. 1 Radiography. Lateral (a) and oblique $(\mathbf{b}, \mathbf{c})$ radiographs of the cervical spine show the sequelae of the flexion-distraction injury centered at $\mathrm{C} 4-\mathrm{C} 5$ with an acute kyphosis (white curved line), mild anterolisthesis of $\mathrm{C} 4$ on $\mathrm{C} 5$, distraction of the posterior disk space (straight black line) and widening of the interspinous distance (curved black line). Both $\mathrm{C} 4$ inferior articular processes are perched on the $\mathrm{C} 5$ superior articular processes (orange lines), and there are small osseous fracture fragments at the anteroinferior endplate of $\mathrm{C} 4$ related to compressive injury (white arrow) and adjacent to the facets related to distraction injury (black arrow). Yellow lines denote normal alignment of the inferior and superior articular processes for comparison

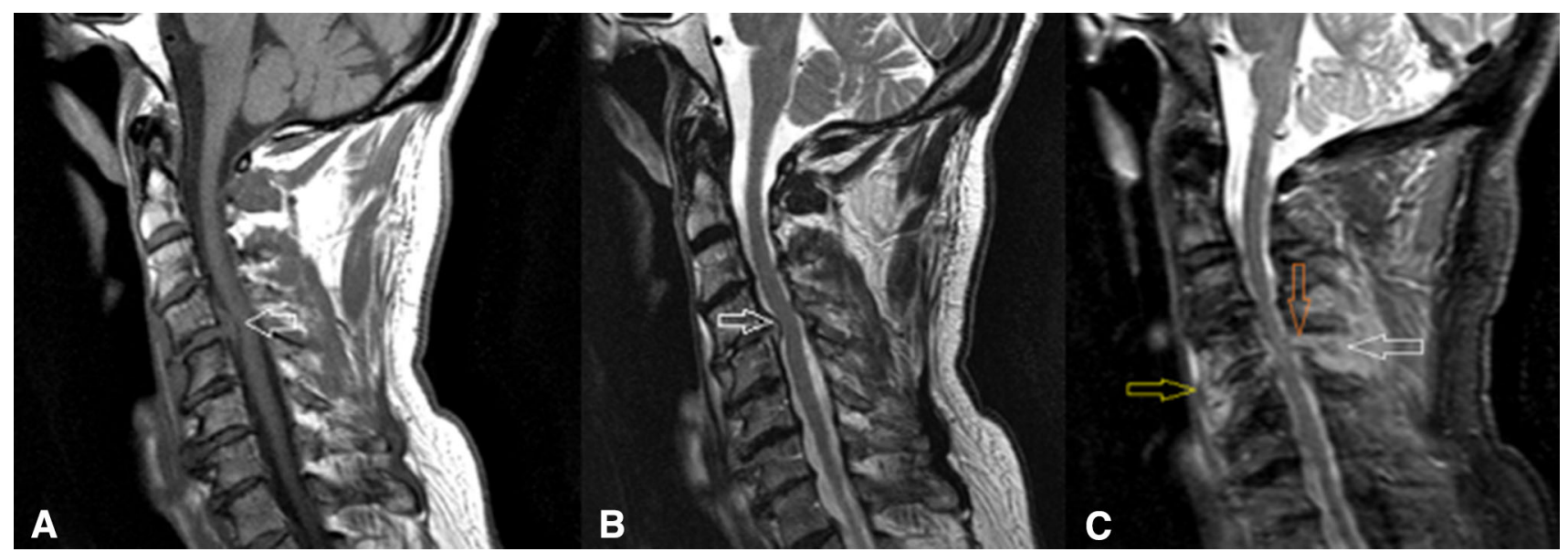

Fig. 2 MRI. Sagittal T1 (a), T2 (b) and Short Tau Inversion Recovery (STIR) (c), MR images of the cervical spine show posterior extrusion of the C4-C5 intervertebral disk (white arrows in $\mathbf{a}, \mathbf{b}$ ). Note the relative high $\mathrm{T} 2$ signal in the intervertebral disk in $\mathbf{b}$ related to the compressive injury. The posterior longitudinal ligament is bowed without clear disruption, and the disk extrusion causes narrowing of the spinal canal, although there is no abnormal cord

integrity of the PLL occurs in $22 \%$ of bilateral facet dislocation injuries [3].

Upon our patient's initial hospital presentation after his fall, he was evaluated by neurosurgery. Reportedly, he had neck pain, but was entirely intact neurologically. Unfortunately, imaging from his initial hospital encounter is not available. He was managed non-operatively in a rigid cervical collar with planned neurosurgical follow-up. After signal (b). c Hyperintense T2 signal posteriorly between the spinous processes (white arrow) and between the laminae (orange arrow) consistent with interspinous and ligamentum flavum ligament injury, respectively, from the distraction injury. There is prevertebral and anterior bone marrow edema at $\mathrm{C} 4-\mathrm{C} 5$ (yellow arrow in $\mathbf{c}$ ) related to compressive injury

discharge from the hospital, he had persistent neck pain, but remained neurologically asymptomatic. He had followup imaging performed 5 weeks after his fall, which showed perched facets (Fig. 1). The vast majority of patients with bilateral perched or jumped facets present with neurological symptoms ranging from radiculopathy to tetraplegia; however, in a few cases such as ours, patients present asymptomatic neurologically. There have been studies 
suggesting that the degree of anterior vertebral displacement correlates with the neurologic status [4], but this is not completely clear. Our patient did have mild anterolisthesis of $\mathrm{C} 4$ on $\mathrm{C} 5$, but he remained neurologically unimpaired. However, his constellation of imaging findings is consistent with a flexion-distraction type injury and cervical spine instability. Treatment options for perched cervical facets include reduction with halo traction and ORIF. However, the ligamentous injury commonly associated with bilateral facet dislocations has a high incidence of redislocation after reduction. Furthermore, bilateral facet dislocations have a high association with disk herniation, and in these instances, traction is not advised since it could exacerbate the herniation and cause neurologic compromise. Therefore, ORIF and ACDF are often indicated. Accordingly, our patient underwent ORIF with ACDF for treatment of his bilateral perched C4-C5 facets, posterior ligamentous injury and disk extrusion causing canal stenosis. Remarkably and thankfully, he maintained normal neurologic status throughout his course, and was discharged after an uneventful postoperative course.

\section{Conclusion}

A rare case of a neurologically intact patient diagnosed with bilateral perched facets 5 weeks after the inciting injury is presented. The injury mechanism and relevant imaging findings are discussed.

\section{Compliance with ethical standards}

Conflict of interest The authors declare that they have no conflict of interest.

Statement of human and animal rights This article does not contain any studies with human participants or animals performed by any of the authors.

Informed consent For this type of study formal consent is waived.

\section{References}

1. Allen BL, Ferguson RL, Lehmann TR, O'Brien RP (1982) A mechanistic classification of closed, indirect fractures and dislocations of the lower cervical spine. Spine 7:1-27

2. Panjabi MM, Simpson AK, Ivancic PC, Pearson AM, Tominaga Y, Yue JJ (2007) Cervical facet joint kinematics during bilateral facet dislocation. Eur Spine J 16:1680-1688

3. Carrino JA, Manton GL, Morrison WB et al (2006) Posterior longitudinal ligament status in cervical spine bilateral facet dislocations. Skelet Radiol 35:510-514

4. O'Connor PA, McCormack O, McCormack J, O'Byrne J (2003) Anterior displacement correlates with neurological impairment in cervical facet dislocations. Int Orthop (SICOT) 27:190-193 